\title{
Erratum: Ab Initio Magneto-Optical Spectrum of Group-IV Vacancy Color Centers in Diamond [Phys. Rev. X 8, 021063 (2018)]
}

\author{
Gergő Thiering $\odot$ and Adam Gali॰
}

(Received 29 May 2020; published 21 July 2020)

DOI: 10.1103/PhysRevX.10.039901

We have found typos in our published paper. We note that none of these issues changes the conclusion or numerical results of the manuscript. We thank Alison E. Rugar from Stanford University for pointing out the typos in Eq. (9) and Appendix C.

Correction to Eq. (8)

The formula in Eq. (8) applies for the rate and not for the lifetime $(\tau)$. The formula of the lifetime is the following:

$$
\tau=\frac{3 \pi \varepsilon_{0}}{n \omega^{3}|\mu|^{2}}
$$

\section{Correction to Tables I and II}

In Table I we introduced some erroneous values. The experimental Huang-Rhys factor $S_{\text {exp }}$ for $\mathrm{SnV}$ did not exist, and we wrote the theoretical value from our calculations. The experimental value for $\mathrm{GeV}$ from Ref. [1] should also be corrected. On the other hand, $S_{\exp }$ values were all correct in Fig. 4 of the paper. The correct values with the corresponding references are collected below.

There was a minor typo for the experimental lifetime $\left(\tau_{\mathrm{PL}}\right)$ in Table II, as the reference for $\mathrm{GeV}(-)$ was used for $\operatorname{SnV}(-)$. Therefore, the footnote of $\sim 5^{\mathrm{c}}$ is Ref. [5] and not Ref. [8].

Correction to Eq. (9) and Appendix C

The $\mu_{B}$ constant is missing from the effective spin Hamiltonian in Eq. (9), and the sign of the third term is positive. We define the $\lambda$ in energy units. Thus, in our paper, it has already been multiplied by Planck's constant $(h)$ in order to convert the units from frequency to energy. We note that the reduction factors $(p, \delta, f)$, and the angular- and spin-momentum operators $\left(\hat{L}_{z}, \hat{S}_{z}\right)$ are dimensionless in Eq. (9),

TABLE I. The calculated zero-phonon-line (ZPL) energies and HR factors $(S)$ for $X \mathrm{~V}(-)$ defects are given and compared to the experimental data $\left(\mathrm{ZPL}_{\text {expt }}\right.$ and $\left.S_{\text {expt }}\right)$. Here, we provide the average ZPL values in $C_{2 h}$ symmetry $\left[\mathrm{ZPL}\left(C_{2 h}\right)\right]$ and within the exact calculation of the dynamic Jahn-Teller (DJT) effect, together with spin-orbit coupling [ZPL (SOC)]. The latter, more accurate method brings our results closer to the experimental values. See text for the explanation.

\begin{tabular}{lcccr}
\hline \hline & $\mathrm{SiV}$ & $\mathrm{GeV}$ & $\mathrm{SnV}$ & $\mathrm{PbV}$ \\
\hline ZPL $\left(C_{2 h}\right)(\mathrm{eV})$ & 1.72 & 2.15 & 2.11 & 2.45 \\
ZPL $(\mathrm{SOC})(\mathrm{eV})$ & 1.70 & 2.12 & 2.09 & 2.40 \\
$S$ & 0.27 & 0.58 & 0.89 & 1.60 \\
ZPL $_{\text {exp }}(\mathrm{eV})$ & $1.68^{\mathrm{a}}$ & $2.06^{\mathrm{b}}$ & $2.00^{\mathrm{c}}$ & \\
$S_{\text {exp }}$ & $0.24^{\mathrm{d}}$ & $0.5^{\mathrm{e}}$ & & \\
\hline \hline
\end{tabular}

${ }^{\mathrm{a}}$ Ref. [2]

${ }^{\mathrm{b}}$ Ref. $[1,3,4]$

${ }^{\mathrm{c}}$ Ref. [5]

${ }^{\mathrm{d}}$ Ref. [6,7]

${ }^{\mathrm{e}}$ Ref. [1]

Published by the American Physical Society under the terms of the Creative Commons Attribution 4.0 International license. Further distribution of this work must maintain attribution to the author(s) and the published articles title, journal citation, and DOI. 
TABLE II. The calculated radiative lifetimes $\left(\tau_{\text {rad }}\right)$ versus the observed photoluminescence lifetimes $\left(\tau_{\mathrm{PL}}\right)$ for $X \mathrm{~V}(-)$ color centers at cryogenic temperatures. We use the experimental ZPL energy where available in the calculation of $\tau_{\text {rad }}$. We note that $\tau_{\mathrm{PL}}$ involves both radiative and nonradiative processes.

\begin{tabular}{lcccc}
\hline \hline & $\mathrm{SiV}(-)$ & $\mathrm{GeV}(-)$ & $\mathrm{SnV}(-)$ & $\mathrm{PbV}(-)$ \\
\hline$\tau_{\text {rad }}(\mathrm{ns})$ & 12.13 & 6.62 & 5.49 & 2.88 \\
$\tau_{\mathrm{PL}}(\mathrm{ns})$ & $1.72^{\mathrm{a}}$ & $\sim 6^{\mathrm{b}}$ & $\sim 5^{\mathrm{c}}$ & \\
\hline \hline
\end{tabular}

${ }^{\mathrm{a}}$ Ref. [9]

${ }^{\mathrm{b}}$ Ref. [8]

${ }^{c}$ Ref. [5]

$$
\hat{H}_{\text {eff }}^{g, u}=\underbrace{-\left(p^{g, u} \lambda_{0}^{g, u}+K_{J T}\right)}_{-\lambda^{g, u}} \hat{L}_{z} \hat{S}_{z}+\mu_{B} \underbrace{p^{g, u} g_{L}^{g, u}}_{f^{g, u}} \hat{L}_{z} B_{z}+\mu_{B} g_{S} \hat{\boldsymbol{S}} \boldsymbol{B}+\mu_{B} \underbrace{2 \delta_{p}^{g, u} g_{L}^{g, u}}_{2 \delta_{f}^{g, u}} \hat{S}_{z} B_{z}+\hat{\Upsilon}_{\text {strain }}
$$

The negative sign originates from the hole picture introduced in Appendix B. Equation (C1) reads correctly as

$$
\hat{H}_{\text {eff }}^{\Gamma}=|\Gamma\rangle\langle\Gamma|\left[p_{\Gamma}^{g, u} \hat{L}_{z}\left(-\lambda_{0}^{g, u} \hat{S}_{z}+\mu_{B} g_{L}^{g, u} B_{z}\right)+\mu_{B} g_{S} \hat{\boldsymbol{S}} \boldsymbol{B}\right] .
$$

Equations (C2) and (C3) contain no errors. Equation (C4) is correct in terms of the signs,

$$
\begin{aligned}
\hat{H}_{\mathrm{eff}}^{g, u} & =\sum_{\Gamma=E_{g, u \frac{1}{2}}, E_{g, u \frac{3}{2}}} \hat{H}_{\mathrm{eff}}^{\Gamma} \\
& =\left(\frac{1}{2}-\hat{L}_{z} \hat{S}_{z}\right) p_{\frac{1}{2}}^{g, u} \hat{L}_{z}\left(-\lambda_{0 g, u} \hat{S}_{z}+\mu_{B} g_{L}^{g, u} B_{z}\right)+\left(\frac{1}{2}+\hat{L}_{z} \hat{S}_{z}\right) p_{\frac{3}{2}}^{g, u} \hat{L}_{z}\left(-\lambda_{0}^{g, u} \hat{S}_{z}+\mu_{B} g_{L}^{g, u} B_{z}\right)+\mu_{B} g_{S} \hat{\boldsymbol{S}} \boldsymbol{B} ;
\end{aligned}
$$

however, Eq. (C5) reads correctly as

$$
\hat{H}_{\text {eff }}^{g, u}=\underbrace{\frac{1}{2}\left(p_{\frac{3}{2}}^{g, u}+p_{\frac{1}{2}}^{g, u}\right)}_{p_{g, u}} \hat{L}_{z}\left(-\lambda_{0 g, u} \hat{S}_{z}+g_{L}^{g, u} B_{z}\right)-\underbrace{\left(p_{\frac{3}{2}}^{g, u}-p_{\frac{1}{2}}^{g, u}\right) \lambda_{0}^{g, u}}_{\text {constant offset }} \underbrace{\hat{L}_{z}^{2} \hat{S}_{z}^{2}}_{\frac{1}{4}}+\underbrace{\left(p_{\frac{3}{2}}^{g, u}-p_{\frac{1}{2}}^{g, u}\right) \mu_{B} g_{L}^{g, u}}_{2 \delta_{g, u}^{f}} \hat{S}_{z} B_{z} \underbrace{L_{z}^{2}}_{1}+\mu_{B} g_{S} \hat{\boldsymbol{S}} \boldsymbol{B} .
$$

Finally, Eq. (C6) is

$$
\hat{H}_{\mathrm{eff}}^{g, u}=-\lambda^{g, u} L_{z} S_{z}+f^{g, u} \mu_{B} L_{z} B_{z}+g_{S} \mu_{B} S \boldsymbol{B}+2 \delta_{f}^{g, u} \mu_{B} S_{z} B_{z} .
$$

These changes do not affect the results, the discussion, or the conclusion of the paper.

As a final remark, Fig. 6 [and Figs. 5(c) and 5(e) in Ref. [10]] can be simulated by the following effective Hamiltonian,

$$
\hat{H}_{\mathrm{eff}}^{g, u} / h=\left[\begin{array}{cccc}
\frac{\lambda^{g, u}}{2}+\left(-\mu_{B} f^{g, u}+\frac{1}{2} \mu_{B} g_{S}+\mu_{B} \delta_{f}^{g, u}\right) B_{z} & 0 & 0 & \frac{1}{2} \mu_{B} g_{S} B_{x} \\
0 & \frac{\lambda^{g, u}}{2}+\left(\mu_{B} f^{g, u}-\frac{1}{2} \mu_{B} g_{S}-\mu_{B} g_{f}^{g, u}\right) B_{z} & \frac{1}{2} \mu_{B} g_{S} B_{x} & 0 \\
0 & \frac{1}{2} \mu_{B} g_{S} B_{x} & -\frac{\lambda x^{g, u}}{2}+\left(\mu_{B} f^{g, u}+\frac{1}{2} \mu_{B} g_{S}+\mu_{B} \delta_{f}^{g, u}\right) B_{z} & 0 \\
\frac{1}{2} \mu_{B} g_{S} B_{x} & 0 & 0 & -\frac{\lambda^{g, u}}{2}+\left(-\mu_{B} f^{g, u}-\frac{1}{2} \mu_{B} g_{S}-\mu_{B} \delta_{f}^{g, u}\right) B_{z}
\end{array}\right] / h,
$$

where we transformed the Hamiltonian presented in Eq. (C5) or Eq. (9) of the main text into a matrix representation. We divided the Hamiltonian by $h$ Planck's constant; thus, the results are in frequency units. The magnetic field is aligned along the $\langle 100\rangle$ direction, which implies $B_{z}=B \sqrt{1 / 3}, B_{x}=B \sqrt{2 / 3}$, and $B_{y}=0$.

Correction to Fig. 5

The signs along the top axis differ from those along the bottom axis in Fig. 5. The signs along the bottom are correct. Here, we show the corrected Fig. 5 with yellow highlighting. 


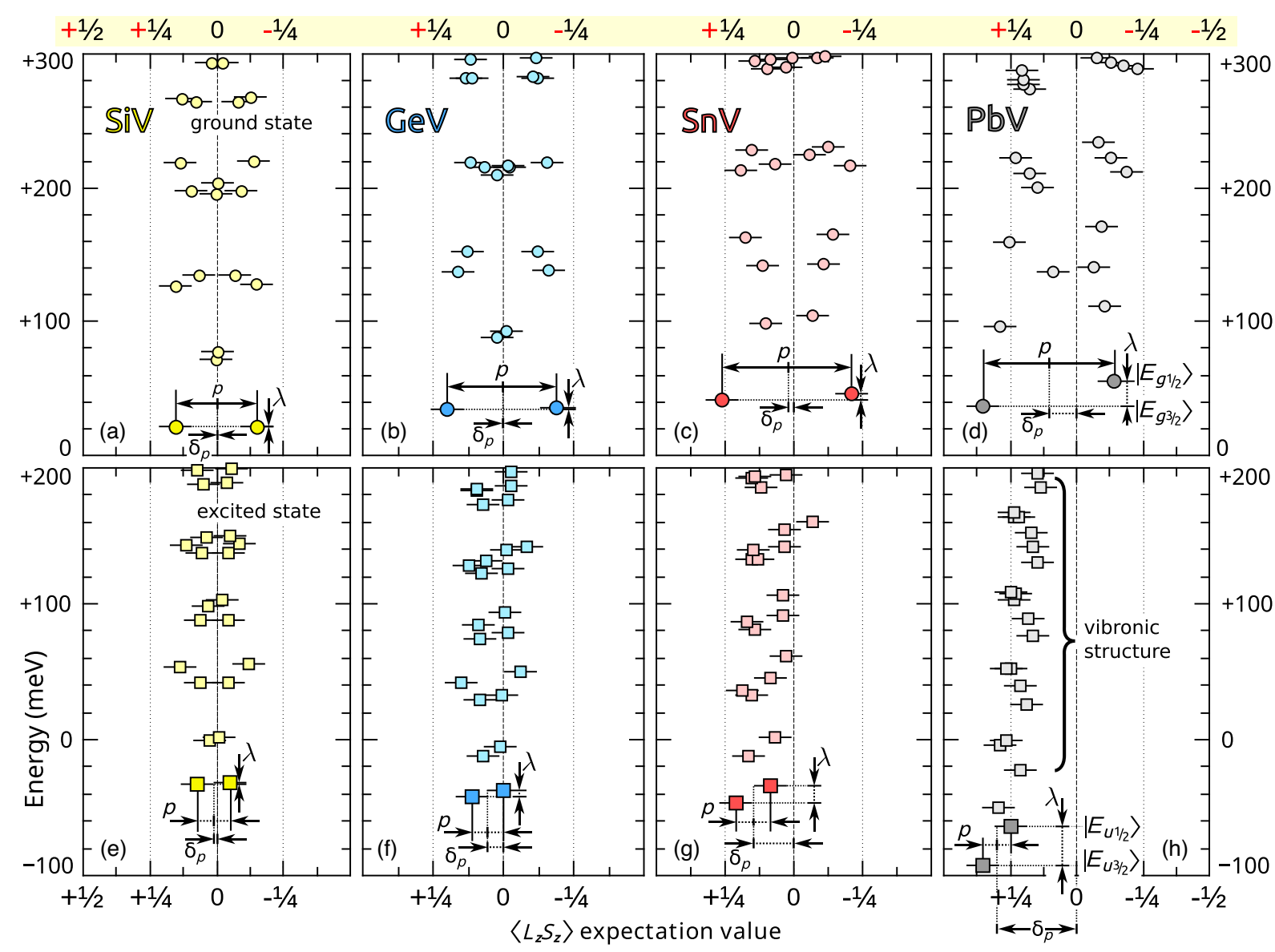

FIG. 5. Calculated eigenvalues of the adjoint DJT [Eq. (4)] and SOC [Eq. (2)] interaction for the XV(-) color centers. The two lowest eigenvalues for each figure correspond to the ${ }^{2} E_{g ; u}$ vibronic $g$ ground states (a-d) and the optically allowed $u$ excited states $(\mathrm{e}-\mathrm{h})$. All of the considered eigenvalues are doubly degenerate in spin dimension because of the Kramers degeneracy. Each state consists of a pure $\uparrow$ or $\downarrow$ spin state; thus, the fourfold degeneracy of ${ }^{2} E_{g, u}$ is fulfilled. We label the energy difference of the two lowest-energy states by $\lambda$, which is directly observed in the fine structure of the ZPL in the PL spectrum known as zero-field splitting. Along the $x$ axis, we depict the eigenvalues with respect to their partially quenched spin-orbit coupling strength $\left\langle L_{z} S_{z}\right\rangle$; thus, one can directly read out the $p$ factors from this figure. Here, $\delta_{p}$ shows deviation of the Ham reduction factors on the $E_{g, u \frac{3}{2}}$ and $E_{g, u \frac{1}{2}}$ states. The larger the $\delta_{p}$, the less accurate the treatment of SOC as a perturbation over DJT. We note that a mirror symmetry at $x=0$ shows up for the ground state of the $\mathrm{SiV}$ center in the entire vibronic spectrum, which demonstrates that SOC can be treated as a perturbation over the JT effect. The systematic left shift at the $x$ axis for the vibronic spectrum of the SnV and PbV defects implies that SOC is comparable to the JT coupling.

[1] Y. N. Palyanov, I. N. Kupriyanov, Y. M. Borzdov, and N. V. Surovtsev, Germanium: A New Catalyst for Diamond Synthesis and a New Optically Active Impurity in Diamond, Sci. Rep. 5, 14789 (2015).

[2] A. Zaitsev, V. Vavilov, and A. Gippius, Cathodoluminescence of Diamond Associated with Silicon Impurity, Sov. Phys. Lab. Inst. Rep. 10, 15 (1981).

[3] E. A. Ekimov, S. G. Lyapin, K. N. Boldyrev, M. V. Kondrin, R. Khmelnitskiy, V. A. Gavva, T. V. Kotereva, and M. N. Popova, Germanium-Vacancy Color Center in Isotopically Enriched Diamonds Synthesized at High Pressures, JETP Lett. 102, 701 (2015).

[4] Y. N. Palyanov, I. N. Kupriyanov, Y. M. Borzdov, A. F. Khokhryakov, and N. V. Surovtsev, High-Pressure Synthesis and Characterization of Ge-Doped Single Crystal Diamond, Cryst. Growth Design 16, 3510 (2016).

[5] T. Iwasaki, Y. Miyamoto, T. Taniguchi, P. Siyushev, M. H. Metsch, F. Jelezko, and M. Hatano, Tin-Vacancy Quantum Emitters in Diamond, Phys. Rev. Lett. 119, 253601 (2017).

[6] A. T. Collins, L. Allers, C. J. Wort, and G. A. Scarsbrook, The Annealing of Radiation Damage in de Beers Colourless CVD Diamond, Diam. Relat. Mater. 3, 932 (1994).

[7] E. Neu, D. Steinmetz, J. Riedrich-Möller, S. Gsell, M. Fischer, M. Schreck, and C. Becher, Single Photon Emission from SiliconVacancy Colour Centres in Chemical Vapour Deposition Nano-Diamonds on Iridium, New J. Phys. 13, 025012 (2011). 
[8] M. K. Bhaskar et al., Quantum Nonlinear Optics with a Germanium-Vacancy Color Center in a Nanoscale Diamond Waveguide, Phys. Rev. Lett. 118, 223603 (2017).

[9] L. Rogers et al., Multiple Intrinsically Identical Single-Photon Emitters in the Solid State, Nat. Commun. 5, 4739 (2014).

[10] A. E. Rugar, C. Dory, S. Sun, and J. Vučković, Characterization of Optical and Spin Properties of Single Tin-Vacancy Centers in Diamond Nanopillars, Phys. Rev. B 99, 205417 (2019). 